\title{
CK8 phosphorylation induced by compressive loads underlies the downregulation of CK8 in human disc degeneration by activating protein kinase $C$
}

\author{
Zhen Sun ${ }^{1,4}$, Yun-Shan Guo ${ }^{1,4}$, Shi-Ju Yan², Zhong-Yuan Wan ${ }^{1}$, Bo Gao ${ }^{1}$, Long Wang ${ }^{1}$, Zhi-Heng Liu' ${ }^{1}$ Yang Gao ${ }^{1}$, \\ Dino Samartzis ${ }^{3}$, Li-Feng Lan', Hai-Qiang Wang ${ }^{1}$ and Zhuo-Jing Luo ${ }^{1}$
}

Cytokeratin 8 (CK8) is a member of the cytokeratins family with multiple functions on the basis of its unique structural hallmark. The aberrant expression of CK8 and its phosphorylation are pertinent with various diseases. We have previously shown that CK8 exists in normal human nucleus pulposus (NP) cells and decreases as the intervertebral disc degenerates. However, the underlying molecular regulatory machinery of CK8 in intervertebral disc degeneration (IDD) has not been clarified. Here, we collected NP samples from patients with idiopathic scoliosis as control and IDD as degenerate groups. We found that CK8 expression decreased in IDD with an increased phosphorylation in degenerate NP cells. Moreover, NP cells were cultured under different compressive load schemes for diverse time duration. We found that compressive loads resulted in phosphorylation and disassembly of CK8 in a time-dependent and degree-dependent manner in vitro. The activation of protein kinase $\mathrm{C}$ was a significant molecular factor contributing to this phenomenon. Taken together, this study is the first to address the molecular mechanisms of CK8 downregulation in NP cells. Importantly, our findings provide clues regarding a molecular link between compressive loads and CK8 alterations, which shed a novel light on the etiology of IDD.

Laboratory Investigation (2013) 93, 1323-1330; doi:10.1038/labinvest.2013.122; published online 28 October 2013

KEYWORDS: compressive load; cytokeratin 8; intervertebral disc degeneration; phosphorylation; protein kinase C

Among the multiple contributing factors of human intervertebral disc degeneration (IDD), mechanical stress is of critical importance given the timely mechanical environment of the disc. ${ }^{1}$ Histologically, the intervertebral disc is composed of an inner gelatinous nucleus pulposus (NP) surrounded by the outer annulus fibrosus (AF). ${ }^{2}$ Normal human NP consists of a large amount of extracellular matrix interspersed by a small number of cells that make up approximately $1 \%$ of the total volume. ${ }^{3}$ This specialized structure is consistent with its function, among which is to provide tremendous stability in the environment of forces and compressive loads. Under this unique mechanical circumstance, the NP cells are capable of responding to compressive loads with altered synthesis rates, content or gene expression for collagens and proteoglycans. ${ }^{4-6}$
Accumulating evidence has shown that cytokeratin 8 (CK8) exists in normal human NP cells and decreases with age. ${ }^{7,8} \mathrm{We}$ also previously found a downregulated CK8 expression with IDD based upon multiple lines of evidence. ${ }^{9} \mathrm{CK} 8$ is a member of the cytokeratins family, which belongs to the intermediate filament proteins of epithelial cells. ${ }^{10}$ Cytokeratins have multiple functions due to their unique structural feature, ie, the maintenance of response to mechanical stress, the regulation of Fas-mediated apoptosis and the modulation of cell size and protein synthesis. ${ }^{11,12}$ Meanwhile, it is noteworthy that the phosphorylation of cytokeratins is an important form to regulate cytokeratin intermediate filament assembly and their dynamic properties. ${ }^{13,14}$ Cytokeratins undergo dynamic phosphorylation under a variety of stress situations. ${ }^{15}$ The aberrant expression of CK8 and its

\footnotetext{
${ }^{1}$ Department of Orthopaedics, Xijing Hospital, Fourth Military Medical University, Xi'an, People's Republic of China; ${ }^{2}$ Department of Orthopaedics, Tangdu Hospital, Fourth Military Medical University, Xi'an, People's Republic of China and ${ }^{3}$ Department of Orthopaedics and Traumatology, University of Hong Kong, Hong Kong, SAR China

Correspondence: Professor H-Q Wang, MD, PhD or Professor Z-J Luo, MD, PhD, Department of Orthopaedics, Xijing Hospital, Fourth Military Medical University, 127 Changle Western Road, Xi'an 710032, People's Republic of China.

E-mail: hqwang@fmmu.edu.cn or zjluo@fmmu.edu.cn

${ }^{4}$ These authors contributed equally to this work.

Received 6 July 2013; revised 21 August 2013; accepted 23 September 2013
} 
disassembly are found to be associated with various diseases. ${ }^{16,17}$ Given that CK8 is crucially involved in mechanical stimulation, as well as disorders characterized by abnormal apoptotic pathways, we assumed that CK8 might have a role in the process of IDD. To date, current studies have only demonstrated the distribution tendency of CK8 in discs in terms of NP origin and development; the underlying molecular regulation of CK8 in IDD has not been clarified.

Accordingly, the current study aimed at addressing the phosphorylation of CK8 in human NP cells. First, we found a decreased CK8 expression while phosphorylatied CK8 was upregulated in degenerate NP cells. Second, we demonstrated that the phosphorylation and disassembly of CK8 were mediated by compressive load with the activation of protein kinase $\mathrm{C}$ (PKC), the chief of which was $\mathrm{PKC}-\varepsilon$ in vitro.

\section{MATERIALS AND METHODS Tissue Collection}

The study was approved by our Institutional Ethics Review Board (No. 20090611-3). Human NP samples and MRI data were collected as described previously. Briefly, written informed consents were obtained from each patient. NP tissues were obtained from patients with idiopathic scoliosis as control group ( $n=8$; average age 19.5 (range 16-24) years) and IDD as degenerate group ( $n=8$ average age 30.5 (range 23-41) years). Idiopathic scoliosis disc specimens were classified as grade II, and IDD discs were classified as grade IV according to Pfirrmann's grading system. ${ }^{18}$

\section{Human NP Cells Isolation and Compressive Load Cultures}

Specimens from patients were obtained within $2 \mathrm{~h}$ after discectomy. NP tissues were separated from the AF using a stereotaxic microscope carefully and washed with phosphatebuffered saline (PBS) to eliminate contamination and blood. Specimens were digested for $40 \mathrm{~min}$ in $0.2 \%$ pronase and washed with PBS. Then specimens were incubated in $0.25 \%$ type II collagenase at $37^{\circ} \mathrm{C}$ under gentle agitation. After $4 \mathrm{~h}$, a $40-\mu \mathrm{m}$ cell strainer was used to remove remaining tissue debris. Freshly isolated NP cells were obtained after centrifugation at $200 \mathrm{~g}$ for $8 \mathrm{~min}$. The culture medium was changed twice a week. Primarily, cultured NP cells (passage 1) were used for the following steps.

NP cells from patients with idiopathic scoliosis were cultured in the compressive load environment. Briefly, NP cell samples were subjected to compressive stress environment in a compression culture chamber (Taikang Biological Technology, Xi'an, China), which was linked with a high pressure gas cylinder. Controllable compressive stress was applied to the samples for 4,24 and $48 \mathrm{~h}$ at $3 \mathrm{Mpa}$ or at 0.3 , 1.0 and $3.0 \mathrm{MPa}$ for $48 \mathrm{~h} \cdot{ }^{19-21}$ The culture chamber works with compressed gas from the cylinder to the culture dishes, leading to compression of fluid media to the NP cells under controlled pressure. Cells cultured at a similar condition without compression loads were used as control.

\section{Protein Isolation and Immunoblotting}

NP cells from the IDD group and control group were used in this step. In addition, to identify the stress influence, we performed this step on samples from idiopathic scoliosis following compressive load cultures. NP cells were prepared in SDS buffer, detached from the culture dish with a cell scraper and boiled for 5-10 min. Debris of the cells was removed by centrifugation for $20 \mathrm{~min}$ at $4{ }^{\circ} \mathrm{C}$. The protein concentration was detected by the BCA assay (Sigma, Saint Louis, USA). For separation of Triton X-100-soluble and insoluble fractions, Triton X-100 buffer ( $20 \mathrm{mM}$ Hepes, $100 \mathrm{mM} \mathrm{NaCl}, 5 \mathrm{mM} \mathrm{MgCl} 2,5 \mathrm{mM}$ EGTA, $1 \%$ Triton X-100, $1 \mathrm{mM}$ PMSF, $10 \mu \mathrm{g} / \mathrm{ml}$ leupeptin, $10 \mu \mathrm{g} / \mathrm{ml}$ antipain) was used. TX-100 soluble (supernatant) and insoluble (pellet) fractions were obtained by homogenizing on ice and centrifuged for $15 \mathrm{~min}$ at $15000 \mathrm{~g}\left(4^{\circ} \mathrm{C}\right)$. Both fractions were dissolved either in Laemmli sample buffer or in urea buffer. The samples were separated on $10 \%$ polyacrylamide gels. Equal amounts of proteins were loaded on 10\% SDS-PAGE. Following transfer to nitrocellulose membranes, samples were incubated with the primary antibodies as follows: rabbit monoclonal anti-CK8, mouse monoclonal anti-pK8Ser-23, anti-pK8Ser-73, anti-pK8Ser-431 (Abcam, Cambridge, USA), and mouse monoclonal antibody specific to $\beta$-actin (Sigma, Saint Louis, USA) was used as control. Antibody labeling was identified using goat anti-rabbit or anti-mouse horseradish peroxidase (HRP)-conjugated secondary antibodies (Cell Signaling Technology, Boston, USA) and visualized using enhanced chemiluminescence.

For immunoprecipitation, cells were solubilized in RIPA buffer. After pelleting at $16000 \mathrm{~g}$ for $15 \mathrm{~min}$, cytokeratin was immunoprecipitated from the supernatant using A/G-Sepharose coupled with anti-CK8 antibody. Proteins were solubilized in Laemmli sample buffer and then immunoblotted as described above with the relevant antibodies.

\section{Immunofluorescence Staining of Frozen Specimens and Cultured NP Cells}

NP specimens from the IDD group and control group were used in this step. Specimens were snap-frozen and cut in $25 \mu \mathrm{m}$ sections transversally with a cryostat microtome. The frozen sections were fixed in methanol at $-20^{\circ} \mathrm{C}$ for $30 \mathrm{~min}$. Following blockage with $1 \%$ bovine serum albumin PBS, the samples were incubated with rabbit monoclonal antibody to CK8 $(5 \mu \mathrm{g} / \mathrm{ml})$ (ab32357, Abcam, Cambridge, USA), mouse monoclonal anti-pCK8Ser-23, anti-pCK8Ser-73 and antipCK8Ser- 431 for $12 \mathrm{~h}$ at $4{ }^{\circ} \mathrm{C}$. The samples were washed and incubated with Alexa 488-conjugated goat anti-rabbit secondary antibodies or fluor-conjugated anti-mouse secondary antibodies (Molecular Probes, Eugene, OR, USA) for $30 \mathrm{~min}$ in the dark at room temperature. For doubling staining of $\mathrm{CK} 8$ and pCK8Ser-73, the antibodies mentioned above was used simultaneously with the same procedure and incubated in anti-rabbit and anti-mouse secondary antibodies. As for the NP cells from patients with idiopathic 
scoliosis following compressive loads, cell were grown on culture chamber slides and staining for CK8 and pCK8 were performed in a similar process, as well as for the controls. For DNA counterstain, the samples were incubated in medium containing DAPI (4'-6-diamid-ino-2-phenylindole) after washing with PBS. Slides were visualized using a Leica microscope (Leica, Wetzlar, Germany).

\section{PKC Activity and Translocation Assays}

NP cells following compressive load cultures were used in this step. PKC activity was measured using the SignaTECT Protein Kinase C assay system (Promega, Madison, WI) according to the manufacturer's instruction. Briefly, cell lysates were treated with $\left[\gamma^{-32} \mathrm{P}\right]$ ATP and PKC-biotinylated peptide substrate in substrate buffer at $30^{\circ} \mathrm{C}$ for $5 \mathrm{~min}$ and spotted

Table 1 Human oligonucleotide primers used for real-time quantitative polymerase chain

\begin{tabular}{lll} 
Gene & Forward primer & Reverse primer \\
\hline PKC- $\alpha$ & $5^{\prime}$-GCCGCAGTGTCGTTATGAAAGTA-3' & $5^{\prime}$-GCTCCATGTGTGCCATTCAATTAG-3' \\
PKC- $\varepsilon$ & $5^{\prime}$-TGGCGTGACAACTACCACCTTC-3' & $5^{\prime}$-CCGGCCATCATCTCGTACATC-3' \\
PKC- $\gamma$ & $5^{\prime}$-TGGAGTCCTGCTGTATGAGATGTTG-3' & $5^{\prime}$-CAGTTGTCCATGATGGCTTGA-3' \\
PKC- - & $5^{\prime}$-TTCCGAGCCATGCCAAATC-3' & $5^{\prime}$-ATCACTGCCCGTCCACACTG-3' \\
PKC- $\zeta$ & $5^{\prime}$-CTGGGTGTCCTTATGTTGAGATGA-3' & $5^{\prime}$-GACGTGTGAGGCCTTGACAGA-3' \\
$\beta$-actin & $5^{\prime}$-GCCAGTGGATTCCGTACTGT-3' & $5^{\prime}$-GAGCTTGCTTCCTGATTGC-3' \\
&
\end{tabular}
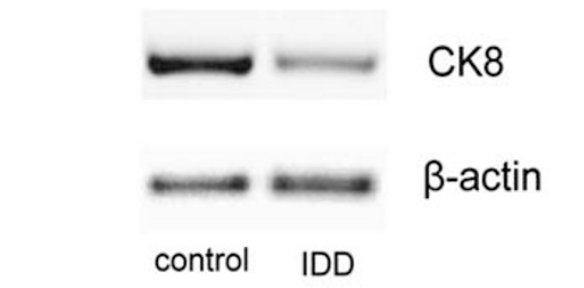

control IDD

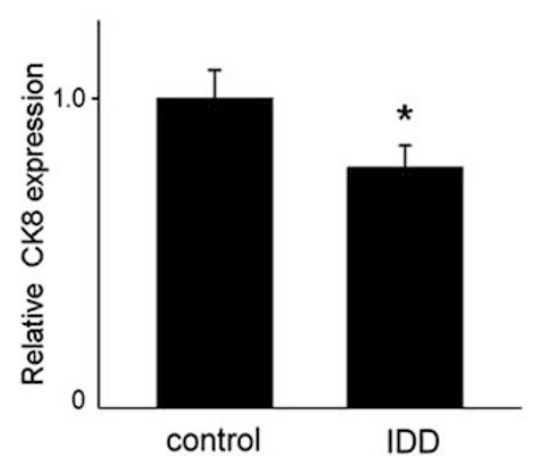

onto SAM biotin capture membrane (Promega, Madison, WI). Subsequently, membranes were washed and incorporated label measured by a scintillation counter. NP cells cultured without compressive load were used as control. In addition, NP cells pretreated with phorbol 12-myristate 13-acetate (PMA) (200 nM) for $24 \mathrm{~h}$ were used as positive control.

\section{Quantitative Real-Time PCR (qRT-PCR) Analysis}

Total RNA in NP cells following compressive load cultures was isolated using TRIzol Reagent (Ambion Invitrogen, Carlsbad, CA, USA) according to the manufacturer's instructions. Reverse transcription to cDNA was performed using a High-Capacity cDNA Archive Kit (ABI, Foster City, CA, USA). RNA concentrations were measured using a NanoDrop instrument (NanoDrop, Wilmington, DE, USA). The levels of mRNA were normalized to GAPDH mRNA controls. All RT reactions, including GAPDH controls, were run in triplicate in a GeneAmp PCR 9700 Thermocycler (ABI). Quantitative real-time PCRs were done on a StepOne Plus device (Applied Biosystems) with SYBR Premix Ex Taq kit (TaKaRa). One microgram of cDNA was amplified at $95^{\circ} \mathrm{C}$ for $15 \mathrm{~s}$ followed by 40 cycles of $95^{\circ} \mathrm{C}, 5 \mathrm{~s}$ and $60^{\circ} \mathrm{C}$ for $30 \mathrm{~s}$. The dissociation curves were performed for all completed SYBR Green reactions to rule out non-specific amplifications and primer dimers. The relative amounts of mRNAs were calculated using the comparative Ct $\left(2^{-\Delta \Delta \mathrm{Ct}}\right)$ method, and samples without compressive load culture were used as
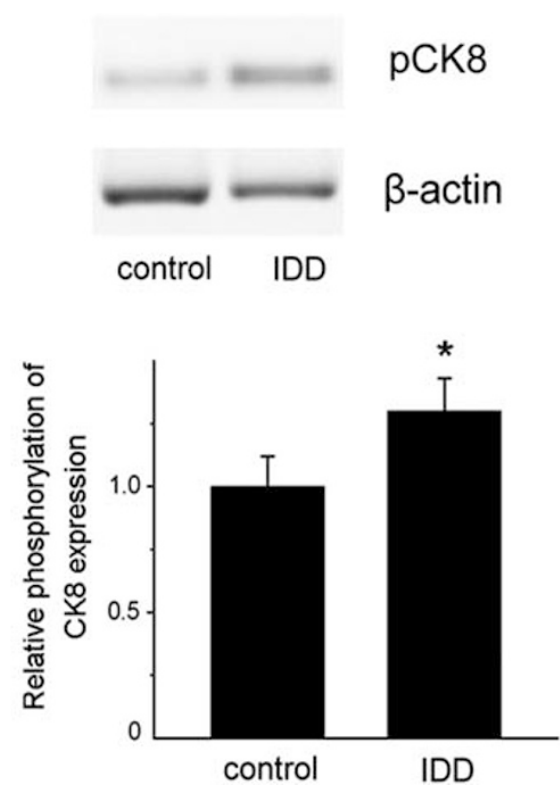

Figure 1 Western blot analysis. Eight NP tissues from patients with idiopathic scoliosis and eight NP tissues from IDD were used as control and IDD groups, respectively. The samples were treated with SDS buffer. Equal amounts of proteins were loaded on 10\% SDS-PAGE. Following transfer to nitrocellulose membranes, samples were incubated with the primary antibodies of anti-CK8, anti-pK8Ser-73 and anti- $\beta$-actin. Antibody labeling was identified using horseradish peroxidase (HRP)-conjugated secondary antibodies. Representative results showed CK8 expression in degenerate disc NP is weaker than that of control, while the expression of CK8 phosphorylation Ser-73 (pCK8) showed a contrary tendency. Quantitative examination showed a similar outcome. Data were performed in triplicate. Error bars represent s.e.m. ${ }^{*} P<0.05$. 

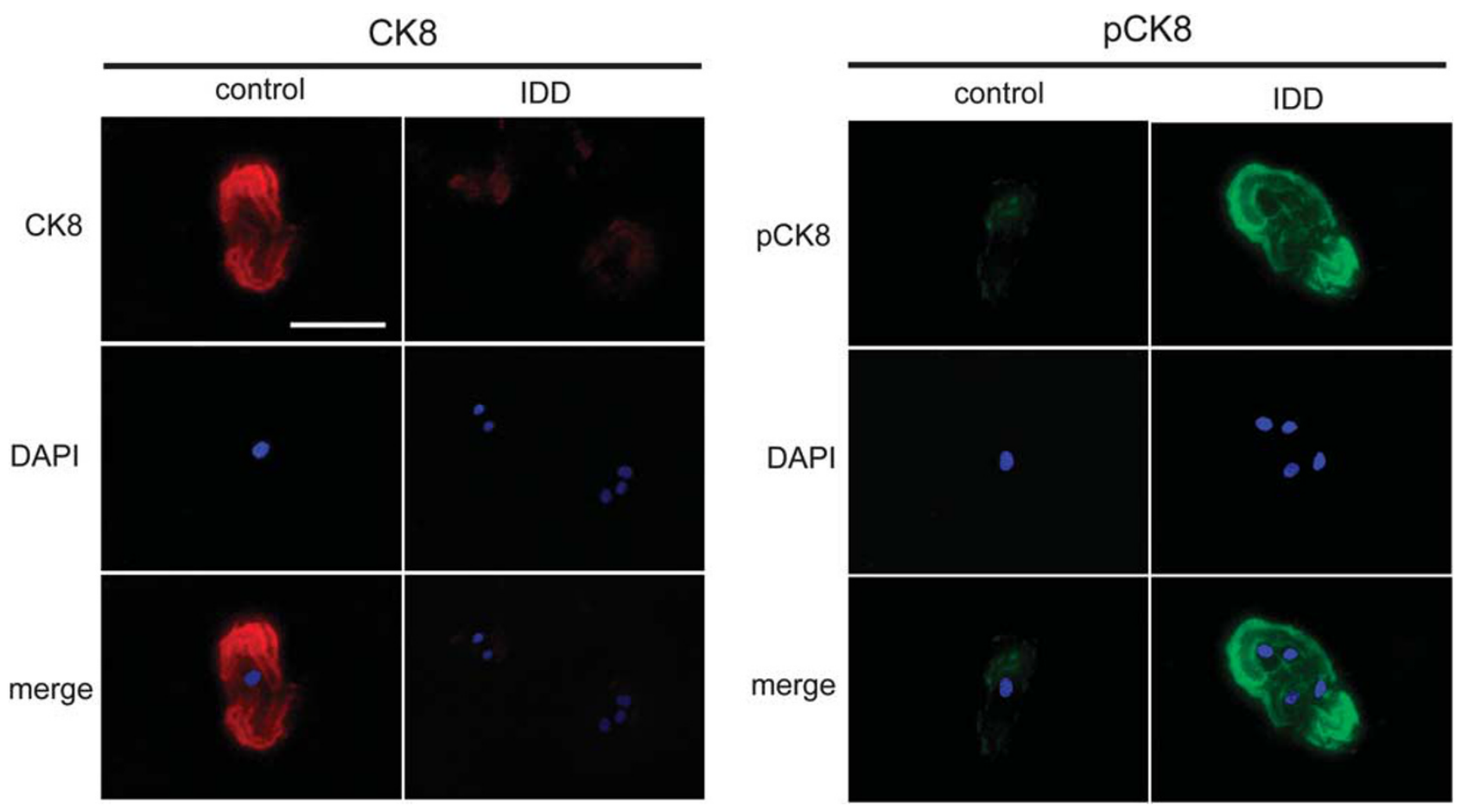

Figure 2 Expression of CK8 and pCK8 (Ser-73) in NP tissues. Specimens from patients with idiopathic scoliosis and IDD were snap-frozen and cut in $25 \mu \mathrm{m}$ transversally with a cryostat microtome. The frozen sections were fixed in methanol at $-20^{\circ} \mathrm{C}$ for 30 min. Following blockage with $1 \%$ bovine serum albumin PBS, the samples were incubated with primary antibodies to CK8 and pCK8Ser- 73 for $12 \mathrm{~h}$ at $4{ }^{\circ} \mathrm{C}$. The samples were washed and incubated with fluor-conjugated secondary antibodies. For DNA counterstain, the samples were incubated in medium containing DAPI (4'-6-diamid-ino2-phenylindole). Expression of CK8 and pCK8 (Ser-73) was detected by fluorescent probes in dark field. CK8 or pCK8 localizes either in the cytoplasm within a single NP cell in control or cell clusters with multiple nuclei in degenerate NP. Bar $=30 \mu \mathrm{m}$.

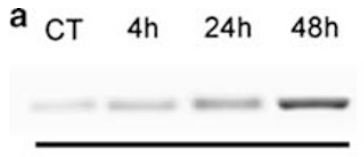

soluble

b

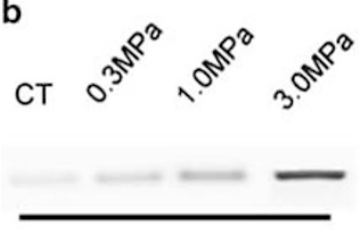

soluble insoluble

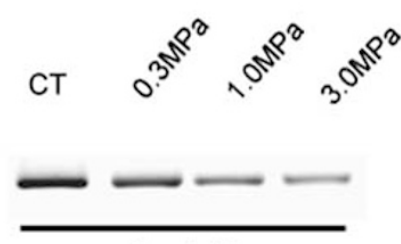

insoluble

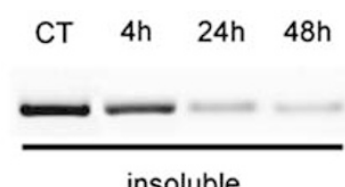

Figure 3 Impact of compressive loads on CK8 solubility. NP cells from eight patients with idiopathic scoliosis were cultured in the compressive load environment for 4,24 and $48 \mathrm{~h}$ at $3 \mathrm{Mpa}$ or at $0.3,1.0$ and $3.0 \mathrm{MPa}$ for $48 \mathrm{~h}$. Cytoskeletal preparations were separated into Triton X-100 (TX-100)-soluble and -insoluble fractions. Equal amounts of protein were separated by $12.5 \%$ SDS-PAGE, transferred to nitrocellulose and immunoblotted with anti-CK8 antibody. (a) Representative immunoblot shows an increased solubility of CK8 upon exposure to compressive load (3.0 MPa) for 4, 24 and $48 \mathrm{~h}$ compared with control (CT).

(b) Representative immunoblot shows an increased solubility of CK8 upon exposure to compressive load $(48 \mathrm{~h})$ at $0.3,1.0$ and $3.0 \mathrm{MPa}$ compared with control (CT). controls. Predesigned primers for PKC- $\alpha$, PKC- $\varepsilon$, PKC $-\gamma$, $\mathrm{PKC}-l, \mathrm{PKC}-\zeta$ and $\beta$-actin as control were designed using the OligoPerfectTM Designer Software (Invitrogen) and purchased from Sangon (Sangon, Shanghai, China) (Table 1).

\section{Statistical Analysis}

Student's $t$-test and ANOVA were used to compare parameters. A $P$ value $<0.05$ was considered statistically significant. The SPSS statistical package (SPSS, Chicago, IL, USA) was used for the statistical analysis.

\section{RESULTS}

\section{CK8 and its Phosphorylation in NP cells}

Western blotting demonstrated a decreased CK8 expression in IDD than that in the control, while the expression of CK8 phosphorylation Ser-73 (pCK8) showed a contrary tendency (Figure 1). Consistent with the western blotting results, immunofluorescence staining of CK8 and pCK8 showed a similar tendency in NP sections (Figure 2). No differences were observed in CK8 phosphorylation (Ser-23 and Ser-431) between the IDD and control group.

\section{Impact of Compressive Load on Phosphorylation of CK8}

Mechanical stimulations, such as shear stress, have been shown to induce phosphorylation and disassembly of keratin 

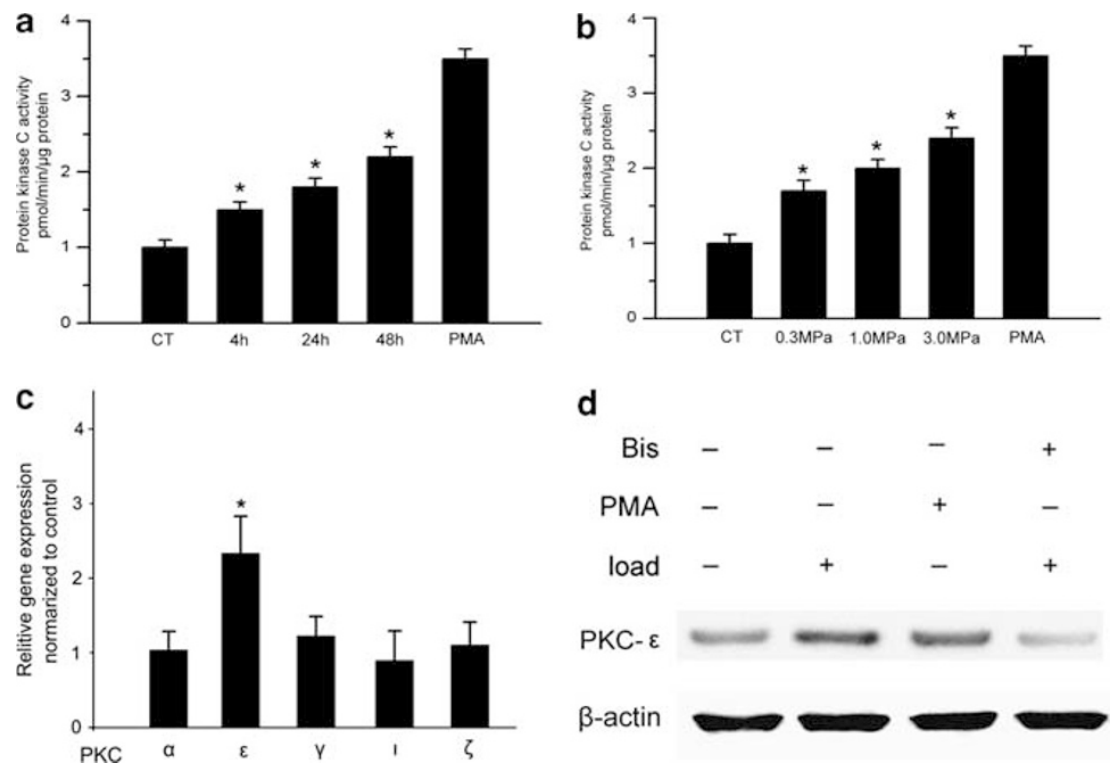

Figure 4 PKC in NP cells. (a, b) NP cells from eight patients with idiopathic scoliosis were exposed to either compressive load environment for 4,24 or $48 \mathrm{~h}$ at $3 \mathrm{MPa}$ or at $0.3,1.0$ and $3.0 \mathrm{MPa}$ for $48 \mathrm{~h}$ and compared with static control cells. Total cell lysates were prepared, and protein kinase $\mathrm{C}$ activity was assayed using the SignaTECT Protein Kinase $C$ assay system. PKC activity increases in a time- and degree-dependent manner exposed to compressive load. Error bars represent s.e.m. ${ }^{*} P<0.05$. (c) qRT-PCR measurement of PKC isoforms (PKC- $\alpha$, PKC $-\varepsilon$, PKC- $\gamma$, PKC $-l$ and PKC- $\zeta$ ) quantifies the mRNA expression in NP cells from eight patients with idiopathic scoliosis after exposure to compressive load at $3.0 \mathrm{MPa}$ for $48 \mathrm{~h}$. The values were quantified and normalized to untreated cells, which was set at 1.0. Error bars represent s.e.m. ${ }^{*} P<0.05$. (d) NP cells from eight patients with idiopathic scoliosis were cultured in compressive load at $3.0 \mathrm{MPa}$ for $48 \mathrm{~h}$. The samples were treated with SDS buffer. Equal amounts of proteins were loaded on $10 \%$ SDS-PAGE. Following transferred to nitrocellulose membranes, samples were incubated with the primary antibodies of anti- PKC- $\varepsilon$ and $\beta$-actin. Antibody labeling was identified using horseradish peroxidase (HRP)-conjugated secondary antibodies. Representative western blot results showed an increased PKC- $\varepsilon$ expression in NP cells after compressive load at $3.0 \mathrm{MPa}$ for $48 \mathrm{~h}$. Treatment with PMA and Bis were used as positive and negative controls.

intermediate filaments in other cells. In the case of NP cells, we assumed that compressive load might similarly be one of the important contributors to the disassembly of CK8. Accordingly, NP cells were cultured in compressive load and then were analyzed for CK8 expression. We used $\beta$-actin for normalization, as it was found unaffected following compressive load cultures. ${ }^{22}$ As shown in Figure 3a, NP cells exposed to compressive load ( $3 \mathrm{MPa}$ ) for 4,24 and $48 \mathrm{~h}$ exhibited a time-dependent decrease in the amount of insoluble (pelletable) CK8, which is consistent with significant increases in the soluble (disassembled) protein. Meanwhile, CK8 assembly depends on the degree of compressive load as shown in Figure 3b. The expression of insoluble CK8 decreased in a load-dependent manner (0.3, 1.0 and 3.0 MPa) in NP cells, whereas the expression of soluble CK8 increased.

\section{Activation of PKC by Compressive Loads}

$\mathrm{NP}$ cells are under a unique pathology circumstance in the compact $\mathrm{AF}$ with high mechanical forces such as compressive loads, which is indicated to activate multiple enzymes. It is known that PKC is closely linked with mechanical stimulation. PKC has an important role in the phosphorylation and disassembly of CKs in many other cells as well. To further investigate the molecular mechanisms of the compressive load effects on phosphorylation and disassembly of CK8, the
PKC activity was measured in NP cells after compressive load cultures. As shown in Figures $4 \mathrm{a}$ and b, NP cells exposed to compressive load showed a time-dependent and degree-dependent activation of PKC compared with control group. Various PKC isoforms exist in NP cells, the chief of which were PKC- $\alpha$, PKC- $\varepsilon$, PKC- $\gamma$, PKC- $-l$ and PKC- $\zeta .{ }^{23}$ To determine the upregulated detailed isoforms after compressive load cultures, qRT-PCR indicated the upregulated expression mRNA of PKC- $\varepsilon$ (Figure 4c). Consistent with the result, western blotting showed an increased expression of PKC- $\varepsilon$ compared with the control. Pre-treatment with bisindolymaleimide (Bis), a PKC inhibitor, prevented the compressive load-mediated increase in PKC- $\varepsilon$. In addition, treatment with a PKC activator, PMA, increased PKC- $\varepsilon$ expression (Figure $4 \mathrm{~d}$ ).

\section{PKC-Mediated CK8 Phosphorylation and Disassembly}

To determine the relationship between PKC and CK8 phosphorylation and disassembly, NP cells treated with PKC activator PMA showed an increased CK8 phosphorylation. Meanwhile, treatment with the PKC inhibitor Bis attenuated the compressive load-mediated increase in CK8 phosphorylation as shown by western blot analysis (Figure 5a). In addition, NP cells treated with Bis prevented the disassembly of CK8 exposed to compressive load (Figure 5b). No obvious 


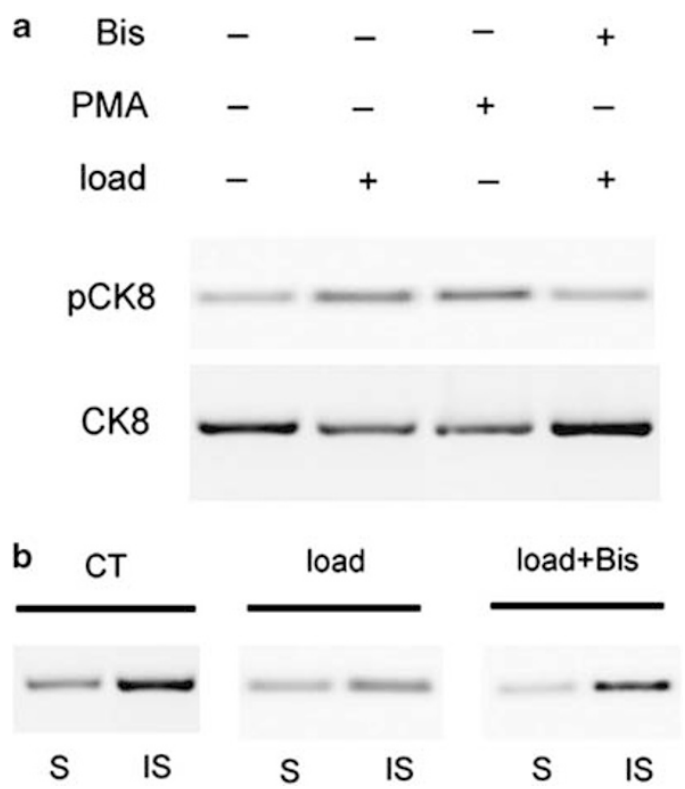

Figure 5 PKC regulates CK8 phosphorylation and assembly in compressive loaded NP cells. NP cells from eight patients with idiopathic scoliosis were cultured in compressive load at $3.0 \mathrm{MPa}$ for $48 \mathrm{~h}$. (a) The samples were treated with SDS buffer. Equal amounts of proteins were loaded on 10\% SDS-PAGE. Following transfer to nitrocellulose membranes, samples were incubated with the primary antibodies of antiCK8, anti-pK8Ser-73 and anti- $\beta$-actin. Antibody labeling was identified using horseradish peroxidase (HRP)-conjugated secondary antibodies. Representative results showed that PKC phosphorylated CK8 in NP cells, which were exposed to $3.0 \mathrm{MPa}$ for $48 \mathrm{~h}$. Treatment with PKC activator PMA showed a similar result, and treatment with Bis, a PKC inhibitor, restrained phosphorylation. (b) NP cell cytoskeletal preparations were separated into Triton X-100-soluble and Triton X-100-insoluble fractions. Equal amounts of protein were separated by A/G-Sepharose, transferred to nitrocellulose and immunoblotted with anti-CK8 antibodies. CK8 was disassembled in compressive load cultures. Treatment with Bis restrained the impact of compressive load. S, Triton X-100-soluble; IS, Triton X-100insoluble.

morphological changes were found in the cells following compressive load cultures. Immunofluorescence of CK8 and pCK8 showed a similar outcome as the western blotting results (Figure 6).

\section{DISCUSSION}

Intermediate filaments such as CK8 are vital component of cell cytoskeleton and have multiple roles in the nucleus and the cytoplasm of eukaryotic cells. In particular, the maintenance of cell shapes under physical stimulation and the regulation of intercellular signals are ascribed to intermediate filaments. Studies show that CK8 expresses in notochord cells during the original development of NP as a marker to classify the origin of NP tissue. ${ }^{24,25}$ In addition, CK8 exists in adult human NP cells and decreases with age or IDD. ${ }^{7,8}$ However, the underlying mechanisms of the downregulation of CK8 are still unidentified. Here, we found that CK8 expression decreases in IDD with phosphorylation in degenerate NP cells. Compressive load is identified as a significant factor contributing to the phosphorylation of $\mathrm{CK} 8$ with the activation of PKC.

The increased expression of phosphorylated CK8 indicates that phosphorylation is one of main causes of CK8 downregulation. Post-translational modifications, especially phosphorylation, are commonly observed in the disassembly and organization of intermediate filaments. It is well established that phosphorylation occurs within the head and tail domains, which are responsible for most of the structural heterogeneity and presumed tissue-specific functions of intermediate filaments. In the case of CK8, a number of in vivo phosphorylation sites have been mapped, which have essential roles in regulating filament assembly and disassembly in vivo. ${ }^{26-29}$

Until now, several lines of evidence have demonstrated that prolonged exposure to hyper-physiological loads is harmful to the disc and consequently contributes to IDD. ${ }^{21,30}$ The structure of NP is unique within the intervertebral disc, which is under tremendous forces and compressive load environment. ${ }^{31,32}$ The exact mechanisms of this effect are complicated, including decreased proteoglycan production, increased apoptosis, the accumulation of inflammatory agents and cell volume changes. ${ }^{33-35}$ In this study, we found that compressive load is a vital factor to mediate the phosphorylation of CK8 in human NP cells. Phosphorylation can result in disassembly of $\mathrm{CK} 8$, leading to the alternations of cytoskeletion and cell signal transduction. As NP cells are in a specific environment with various physical forces, the degradation of CK8 might reduce the cells' force resistance ability and cause a harmful signal transduction, which results in IDD.

To classify the link between compressive loads and CK8 phosphorylation, we found that PKC (the chief of which is PKC- $\varepsilon$ ) is activated in compressive load-cultured NP cells. The activated PKC contributes to the phosphorylation of CK8 and its disassembly. This finding sheds a novel light on the molecular mechanisms of the impact of compressive loads on IDD. In fact, studies have shown that intermediate filaments could be phosphorylated by PKC in many other cells, and this impact could lead to abnormal physiological processes. ${ }^{36,37}$ In the case of NP cells, our results suggest that activated PKC results in the downregulation of CK8, which might be an important contributor to IDD. On the other hand, PKCs, mainly PKC- $\gamma$, can lead to an increase in matrix synthesis and cell proliferation, suggesting a beneficial function of PKC. ${ }^{23}$ However, our findings indicate that PKC might contribute to IDD with the disassembly of cytoskeletion in NP cells. This contradiction might be warranted given that PKC may have a double role in IDD with different isoforms, especially in different physical environment. In addition, our results were consistent with Ellman $e t a l,{ }^{38}$ who found suppression of the PKC- $\delta$ pathway was beneficial in the prevention of IDD. Further studies are needed to confirm whether PKC- $\delta$ has an impact on CK8 expression. 


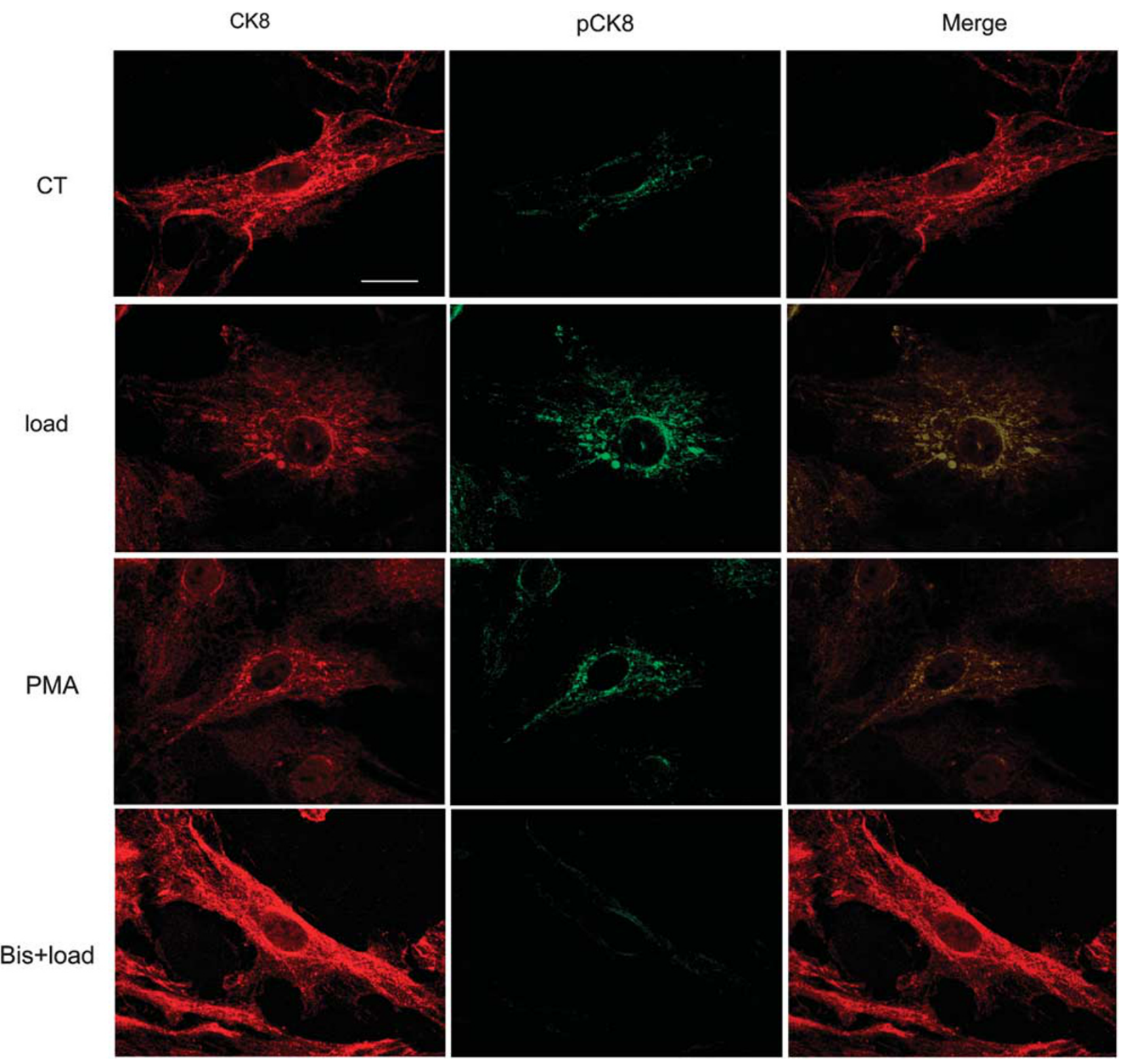

Figure 6 Reorganization of CK8 and pCK8 in NP cells. NP cells from eight patients with idiopathic scoliosis were cultured in compressive load at $3.0 \mathrm{MPa}$ for $48 \mathrm{~h}$. The cells were then fixed in methanol at $-20^{\circ} \mathrm{C}$ for $30 \mathrm{~min}$. Following blockage with $1 \%$ bovine serum albumin PBS, the samples were incubated with primary antibodies to CK8 and pCK8Ser-73 for $12 \mathrm{~h}$ at $4{ }^{\circ} \mathrm{C}$. The samples were washed and incubated with fluor-conjugated secondary antibodies. CK8 was phosphorylated after compressive load cultures compared with the control. Treatment with PMA showed a similar result, and treatment with Bis, a PKC inhibitor, restrained phosphorylation. Bar $=20 \mu \mathrm{m}$.

Although our study sheds new light on the molecular mechanisms of CK8 downregulation in NP cells, there are several drawbacks in the present study. For one, the age of patients with scoliosis as the control group was relatively younger than that of the IDD group. The variation was somewhat inevitable due to the hallmarks of adolescent scoliosis. However, we used unified MRI grading system to clarify the degeneration grades. For another, PKC- $\varepsilon$-specific inhibitor might be more useful to confirm the data. However, NP cell viability might be affected by procedures such as transient permeabilization by saponin to introduce the inhibitor into cells. Moreover, Smolders et $a^{39}$ found that notochordal cells maintain their phenotype when cultured in monolayer for 10 days. As CK8 is expressed in notochordral cells, ${ }^{40,41}$ whether compressive load or PKCs affect CK8 expression especially in the first decade of human life in these cells is unknown.

In conclusion, this is the first study to show the molecular mechanisms of CK8 downregulation in NP cells based upon multiple lines of evidence. As the NP is in a special 
environment with various physical forces, our findings provide clues regarding a molecular link (PKC) between compressive load and alterations in $\mathrm{CK} 8$, which laid a foundation in the study of IDD pathology.

\section{ACKNOWLEDGMENTS}

This work was supported by the Chinese National Natural Science Foundation Grants (No.30901509, No.81270028 and No.81171747).

\section{DISCLOSURE/CONFLICT OF INTEREST}

The authors declare no conflict of interest.

1. Macfarlane GJ, Thomas E, Croft PR, et al. Predictors of early improvement in low back pain amongst consulters to general practice: the influence of pre-morbid and episode-related factors. Pain 1999;80:113-119.

2. Mirza SK, White 3rd AA. Anatomy of intervertebral disc and pathophysiology of herniated disc disease. J Clin Laser Med Surg 1995:13:131-142.

3. Chelberg MK, Banks GM, Geiger DF, et al. Identification of heterogeneous cell populations in normal human intervertebral disc. J Anat 1995;186(Pt 1):43-53.

4. Hutton WC, Toribatake $Y$, Elmer WA, et al. The effect of compressive force applied to the intervertebral disc in vivo. A study of proteoglycans and collagen. Spine 1998;23:2524-2537.

5. Chen J, Yan W, Setton LA. Static compression induces zonal-specific changes in gene expression for extracellular matrix and cytoskeletal proteins in intervertebral disc cells in vitro. Matrix Biol 2004;22: 573-583.

6. Huang $M$, Wang $\mathrm{HQ}$, Zhang $\mathrm{Q}$, et al. Alterations of ADAMTSs and TIMP3 in human nucleus pulposus cells subjected to compressive load: Implications in the pathogenesis of human intervertebral disc degeneration. J Orthop Res 2012;30:267-273.

7. Stosiek P, Kasper M, Karsten U. Expression of cytokeratin and vimentin in nucleus pulposus cells. Differentiation 1988;39:78-81.

8. Weiler $C$, Nerlich AG, Schaaf $R$, et al. Immunohistochemical identification of notochordal markers in cells in the aging human lumbar intervertebral disc. Eur Spine J 2010;19:1761-1770.

9. Sun $Z$, Wang $H Q$, Liu $Z H$, et al. Down-regulated CK8 expression in human intervertebral disc degeneration. Int J Med Sci 2013;10: 948-956.

10. Strnad P, Stumptner C, Zatloukal K, et al. Intermediate filament cytoskeleton of the liver in health and disease. Histochem Cell Biol 2008;129:735-749.

11. Galarneau L, Loranger A, Gilbert $\mathrm{S}$, et al. Keratins modulate hepatic cell adhesion, size and G1/S transition. Exp Cell Res 2007;313:179-194.

12. Fois $\mathrm{G}$, Weimer $\mathrm{M}$, Busch $\mathrm{T}$, et al. Effects of keratin phosphorylation on the mechanical properties of keratin filaments in living cells. FASEB $J$ 2013;27:1322-1329.

13. Ku NO, Michie SA, Soetikno RM, et al. Susceptibility to hepatotoxicity in transgenic mice that express a dominant-negative human keratin 18 mutant. J Clin Invest 1996;98:1034-1046.

14. Eriksson JE, Brautigan DL, Vallee R, et al. Cytoskeletal integrity in interphase cells requires protein phosphatase activity. Proc Natl Acad Sci USA 1992;89:11093-11097.

15. Omary MB, Ku NO, Tao GZ, et al. 'Heads and tails' of intermediate filament phosphorylation: multiple sites and functional insights. Trends Biochem Sci 2006:31:383-394.

16. Kakehashi A, Kato A, Inoue $M$, et al. Cytokeratin $8 / 18$ as a new marker of mouse liver preneoplastic lesions. Toxicol Appl Pharmacol 2010;242:47-55.

17. Gires $\mathrm{O}$, Andratschke $\mathrm{M}$, Schmitt B, et al. Cytokeratin 8 associates with the external leaflet of plasma membranes in tumour cells. Biochem Biophys Res Commun 2005;328:1154-1162.

18. Pfirrmann CW, Metzdorf A, Zanetti M, et al. Magnetic resonance classification of lumbar intervertebral disc degeneration. Spine 2001;26: 1873-1878.
19. Handa $\mathrm{T}$, Ishihara $\mathrm{H}$, Ohshima $\mathrm{H}$, et al. Effects of hydrostatic pressure on matrix synthesis and matrix metalloproteinase production in the human lumbar intervertebral disc. Spine 1997;22:1085-1091.

20. Liu GZ, Ishihara H, Osada R, et al. Nitric oxide mediates the change of proteoglycan synthesis in the human lumbar intervertebral disc in response to hydrostatic pressure. Spine 2001;26:134-141.

21. Chan SC, Ferguson SJ, Gantenbein-Ritter B. The effects of dynamic loading on the intervertebral disc. Eur Spine J 2011;20:1796-1812.

22. Durrant LA, Archer CW, Benjamin M, et al. Organisation of the chondrocyte cytoskeleton and its response to changing mechanical conditions in organ culture. J Anat 1999;194(Pt 3):343-353.

23. Arai F, Hiyama A, Sakai D, et al. The expression and role of non-canonical (PKC) signaling in nucleus pulposus cell metabolism. J Orthop Res 2012;30:1478-1485.

24. Gilson A, Dreger M, Urban JP. Differential expression level of cytokeratin 8 in cells of the bovine nucleus pulposus complicates the search for specific intervertebral disc cell markers. Arthritis Res Ther 2010;12:R24.

25. Risbud MV, Shapiro IM. Notochordal cells in the adult intervertebral disc: new perspective on an old question. Crit Rev Eukaryot Gene Expr 2011;21:29-41.

26. Falconer IR, Yeung DS. Cytoskeletal changes in hepatocytes induced by Microcystis toxins and their relation to hyperphosphorylation of cell proteins. Chem Biol Interact 1992;81:181-196.

27. Toivola DM, Omary MB, Ku NO, et al. Protein phosphatase inhibition in normal and keratin 8/18 assembly-incompetent mouse strains supports a functional role of keratin intermediate filaments in preserving hepatocyte integrity. Hepatology 1998;28:116-128.

28. Moch $M$, Herberich $G$, Aach $T$, et al. Measuring the regulation of keratin filament network dynamics. Proc Natl Acad Sci USA 2013;110: 10664-10669.

29. Jaitovich $\mathrm{A}$, Mehta $\mathrm{S}, \mathrm{Na} \mathrm{N}$, et al. Ubiquitin-proteasome-mediated degradation of keratin intermediate filaments in mechanically stimulated A549 cells. J Biol Chem 2008;283:25348-25355.

30. Zhu Q, Jackson AR, Gu WY. Cell viability in intervertebral disc under various nutritional and dynamic loading conditions: $3 \mathrm{~d}$ finite element analysis. J Biomech 2012;45:2769-2777.

31. Hsieh AH, Twomey JD. Cellular mechanobiology of the intervertebral disc: new directions and approaches. J Biomech 2010;43:137-145.

32. Roberts S, Evans $\mathrm{H}$, Trivedi J, et al. Histology and pathology of the human intervertebral disc. J Bone Joint Surg 2006;88(Suppl 2):10-14.

33. Benallaoua $M$, Richette $P$, Francois $M$, et al. Modulation of proteoglycan production by cyclic tensile stretch in intervertebral disc cells through a post-translational mechanism. Biorheology 2006;43:303-310.

34. Rannou F, Lee TS, Zhou RH, et al. Intervertebral disc degeneration: the role of the mitochondrial pathway in annulus fibrosus cell apoptosis induced by overload. Am J Pathol 2004;164:915-924.

35. Hall AC. Volume-sensitive taurine transport in bovine articular chondrocytes. J Physiol 1995;484(Pt 3):755-766.

36. Omary MB, Baxter GT, Chou CF, et al. PKC epsilon-related kinase associates with and phosphorylates cytokeratin 8 and 18. J Cell Biol 1992;117:583-593.

37. Huang $\mathrm{X}, \mathrm{Li} \mathrm{J}$, Foster $\mathrm{D}$, et al. Protein kinase C-mediated desmin phosphorylation is related to myofibril disarray in cardiomyopathic hamster heart. Exp Biol Med 2002;227:1039-1046.

38. Ellman MB, Kim JS, An HS, et al. The pathophysiologic role of the protein kinase Cdelta pathway in the intervertebral discs of rabbits and mice: in vitro, ex vivo, and in vivo studies. Arthritis Rheum 2012;64:1950-1959.

39. Smolders LA, Meij BP, Riemers FM, et al. Canonical Wnt signaling in the notochordal cell is upregulated in early intervertebral disk degeneration. J Orthop Res 2012;30:950-957.

40. Gotz W, Kasper M, Fischer G, et al. Intermediate filament typing of the human embryonic and fetal notochord. Cell Tissue Res 1995;280: 455-462.

41. Sakai D, Nakai T, Mochida J, et al. Differential phenotype of intervertebral disc cells: microarray and immunohistochemical analysis of canine nucleus pulposus and anulus fibrosus. Spine 2009;34:1448-1456. 